\title{
FACTORES BIOPSICOSOCIALES CORRELACIONADOS CON LA INSATISFACCIÓN DE LA IMAGEN CORPORAL EN ADOLESCENTES MEXICANOS
}

\author{
Yeyetsy G. Ordóñez-Azuara ${ }^{*}$, Nora L. Vázquez-Azuara ${ }^{3}$, Raúl F. Gutiérrez-Herrera ${ }^{2}$, Rosalba Mendoza-Rivera ${ }^{4}$, \\ Héctor M. Riquelme-Heras ${ }^{1}$ y Héctor G. González-Contreras ${ }^{5}$ \\ ${ }^{1}$ Médica familiar, profesor/a del Departamento de Medicina Familiar; ${ }^{2}$ Médico familiar, jefe del Departamento de Medicina Familiar. \\ Universidad Autónoma de Nuevo León, Monterrey, NL.; ${ }^{3}$ Maestra en Investigación Clínica, Médica familiar adscrita a la Unidad de Medicina \\ Familiar 66; ${ }^{4}$ Médica familiar, coordinadora clínica de Educación e Investigación en Salud, Unidad de Medicina Familiar 66; ${ }^{5}$ Licenciado en \\ Estadística, Coordinación de Competitividad y Capacitación, Delegación Veracruz Norte. IMSS, Xalapa, Ver. México
}

RESUMEN: Objetivo: Determinar los factores biopsicosociales correlacionados con la insatisfacción de la imagen corporal en adolescentes. Material y métodos: Estudio prospectivo, transversal y comparativo (correlacional) de 437 adolescentes, seleccionados por muestreo no probabilístico por conveniencia, que fueron divididos usando la escala de Stunkard de acuerdo a su satisfacción o insatisfacción de su imagen corporal. Se utilizó un cuestionario de características sociodemográficas, Kidscreen-52 y medición de peso y talla. Se hizo el análisis estadístico usando medidas de tendencia central, pruebas de chi cuadrada y U de Mann Whitney, y coeficiente de correlación de Spearman. Resultados: Hubo un $69 \%$ de adolescentes insatisfechos con su imagen. Se reportó significancia estadística $(\mathrm{p}<0.05)$ en la insatisfacción con los factores biológicos como el índice de masa corporal (IMC) y de los factores psicosociales, en las dimensiones: bienestar físico, estado de ánimo, autopercepción y relación con los amigos de acuerdo al instrumento usado. Conclusiones: Los factores biopsicosociales correlacionados con la insatisfacción de la imagen corporal en los adolescentes de una población mexicana son: IMC (positiva)
BIOPSYCHOSOCIAL FACTORS CORRELATED WITH BODY IMAGE DISSATISFACTION AMONG MEXICAN ADOLESCENTS

ABSTRACT: Objective: Determine the correlation of biopsychosocial factors with dissatisfaction of the body image in adolescents. Material and methods: This was a prospective, cross-sectional, comparative (correlational) study of 437 adolescents, selected using a non-probabilistic convenience sampling, who were divided using the Stunkard scale according to their body image satisfaction or dissatisfaction; a questionnaire of sociodemographic characteristics, Kidscreen 52 and measurement of weight and height was used. A statistical analysis was performed using central measures of tendency, the chi square test, the Mann Whitney-U test, and Spearman's correlation coefficient. Results: A total of $69 \%$ of adolescents were dissatisfied with their image. Statistical significance in dissatisfaction $(\mathrm{p}<0.05)$ was reported with biological factors such as body mass index and psychosocial factors in the dimensions physical well-being, mood, self-perception and relationship with friends, according to the instrument used. Conclusions: The biopsychosocial factors that correlate with dissatisfaction of body image are: BMI (positive) and physical well-being, mood, self-per-

\author{
Correspondencia: \\ *Yeyetsy G. Ordóñez-Azuara \\ Departamento de Medicina Familiar \\ Universidad Autónoma de Nuevo León \\ Edificio Rodrigo Barragán \\ Hospital Universitario Dr. José E. González \\ Av. Madero y Gonzalitos \\ Col. Mitras Centro \\ C.P. 64460, Monterrey, NLe., México \\ E-mail:yeyetsy_10@hotmail.com
}

Fecha de recepción: 03-04-2018

Fecha de aceptación: 18-06-2018

doi:10.24875/RMF.18000077 
y bienestar físico, estado de ánimo, autopercepción y relación entre pares/amigos (negativa).

Palabras clave: Imagen corporal. Adolescentes. Factores biopsicosociales. ception and relationship with peers/friends (negative). (Rev Mex Med Fam. 2018;5:75-82)

Corresponding author: Yeyetsy G. Ordóñez-Azuara yeyetsy_10@hotmail.com

Key words: Imagen corporal. Adolescentes. Factores biopsicosociales.

\section{INTRODUCCIÓN}

La imagen corporal es la que se forma en la mente acerca del propio cuerpo, considerándose un constructo multifacético de la percepción, los pensamientos y los sentimientos hacia la forma y estructura corporal. De modo que puede haber satisfacción cuando la valoración de la percepción resulta positiva e insatisfacción de la imagen corporal en caso de que la imagen formada en la mente ocasione al sujeto inconformidad con el cuerpo o con alguna de sus partes ${ }^{1-3}$.

Los grupos de población que mayormente están insatisfechos con su imagen son aquellas personas que por distintas circunstancias han experimentado cambios corporales (embarazadas, obesos, adolescentes) o que por motivos profesionales se les imponen estándares corporales (modelos, bailarinas, determinados deportistas). En la actualidad, la insatisfacción de la imagen corporal se ha vuelto un problema mundial moderno de salud: por su elevada incidencia, por ser un factor de riesgo para el desarrollo de trastornos de la conducta alimentaria y por predisponer a conductas de riesgo sobre la salud, como lo es el inicio de tratamientos estéticos de forma reiterada y con demandas irracionales, entre otras ${ }^{4-7}$.

Se han descrito factores predisponentes y de mantenimiento que confluyen en la formación de una imagen corporal negativa en los adolescentes. De los factores biológicos destacan la edad, el sexo, la etnia y el IMC, y de los factores psicosociales, el autoconcepto, la autoestima, las burlas por parte de otros adolescentes, la influencia del entorno familiar, las relaciones sociales, la comparación entre pares y el entorno cultural ${ }^{8-12}$.
De acuerdo a la proyección de la población estimada para el año 2015, los adolescentes son el grupo etario predominante en México, por lo que deben ser considerados una prioridad en el primer nivel de atención en salud, ya que este padecimiento puede mermar su calidad de vida y por ende causar repercusiones socioeconómicas en la nación ${ }^{13-18}$.

Estudios realizados en adolescentes mexicanos han reportado que al menos dos terceras partes de la población estudiada estaba insatisfecha con su imagen, de tal manera que determinar los factores biopsicosociales correlacionados con la insatisfacción de la imagen corporal en adolescentes mexicanos es un eje fundamental de investigación, objetivo que en este trabajo fue determinado a través de un análisis comparativo que permitió establecer las bases para posteriores estudios de causalidad y también se dictaron los factores biopsicosociales relacionados con la insatisfacción de la imagen corporal sobre los cuales es necesario incidir, a través de intervenciones específicas, para prevenir conductas de riesgo $y$, desde una perspectiva sistémica, beneficiar de manera integral la salud de la población ${ }^{12-15}$.

\section{MATERIAL Y MÉTODOS}

Éste es un estudio prospectivo, transversal y comparativo en adolescentes de una secundaria pública mexicana realizado en el periodo de junio a julio de 2015.

\section{Participantes}

Se incluyeron 437 adolescentes inscritos en la secundaria, entre 12 y 17 años de edad, hombres y mujeres, con satisfacción o insatisfacción de la imagen corporal y cuyo padre/madre o tutor otorgó consentimiento 
por escrito. Se excluyeron los adolescentes con alguna enfermedad cognitiva y los que no aceptaron su participación en la prueba, y como criterios de eliminación: adolescentes que no asistieron el día de la prueba y formatos de encuestas con llenado incompleto.

\section{Muestra}

De una población total de 1,526 adolescentes de una secundaria federal, el tamaño muestral se calculó utilizando una fórmula para estimación de proporciones, con un nivel de confianza del $95 \%$ y un error de estimación del $4 \%$, con una proporción del $65 \%$ (posibles alumnos con insatisfacción de la imagen corporal de acuerdo a un estudio previo en adolescentes de esta población mexicana realizado en 2013) ${ }^{15}$, obteniendo así que 403 adolescentes era el número mínimo necesario de participantes para el estudio; sin embargo, para compensar pérdidas por encuestas incompletas, se decidió utilizar un número total de 437 . La técnica de muestreo utilizada fue un muestreo no probabilístico por conveniencia, dado que se estudiaron solamente los alumnos cuyos padres o tutores aceptaron la participación.

\section{Variables e instrumentos de medición}

Las variables estudiadas fueron la satisfacción/insatisfacción de la imagen corporal y los factores biopsicosociales, que se dividieron en factores biológicos y psicosociales.

La percepción de la imagen corporal fue obtenida por medio de la escala de las nueve siluetas propuesta por Stunkard (1983), permitiendo clasificar inicialmente a los adolescentes en satisfechos e insatisfechos, para luego ordenarlos de acuerdo con el grado de insatisfacción. La escala visual de la satisfacción corporal propuesta por Stunkard, et al. se compone de nueve siluetas que cubren un continuo de peso, que va desde una silueta muy delgada hasta una obesa, pasando por una silueta de peso normal. La satisfacción o insatisfacción de la imagen corporal se obtiene a partir de la diferencia entre la figura considerada como la actual y la ideal, donde se interpretarán como insatisfacción de la imagen corporal las diferencias positivas y las negativas (cuanto mayor sea la diferencia, mayor es el grado de insatisfacción). Una diferencia igual a cero significará satisfacción con la imagen corporal. De esta escala se reporta en la bibliografía un $\alpha$ de Cronbach (AC) de $0.89^{15,16}$.

Se consideraron factores biológicos: el sexo y la edad, que se determinaron por el cuestionario de características sociodemográficas, y el IMC, que se determinó por el cálculo obtenido con el peso y talla medidos y clasificados según los percentiles del Centro para el Control y la Prevención de Enfermedades.

Los factores psicosociales considerados fueron: el bienestar físico, el bienestar psicológico, el estado de ánimo, la autopercepción, la autonomía, la relación con los padres/familia, los recursos económicos, la relación con los amigos/pares, el entorno escolar y la aceptación social/bullying, que fueron determinados por el cuestionario Kidscreen-52, un instrumento de autorreporte de salud y bienestar en niños y adolescentes de 8 a 18 años. El cuestionario mide 10 dimensiones de calidad de vida relacionada con la salud (CVRS) y justamente mide los factores que frecuentemente se describen y vinculan con la insatisfacción de la imagen corporal, tiene buena validez y fiabilidad para cada dimensión, reportando un AC de 0.81 para el global de calidad de vida y un AC de 0.80 en su mayoría para cada dimensión ${ }^{17-19}$ :

- Bienestar físico (AC de 0.77): con cinco ítems, recoge los niveles de actividad física del chico/a y la sensación de energía y de estar físicamente en forma.

- Bienestar psicológico (AC de 0.85): con seis ítems, son las emociones positivas y la satisfacción con la vida.

- Estado de ánimo y emociones (AC de 0.84 ): con siete ítems, son las experiencias negativas, la sensación de depresión y el estrés.

- Autopercepción (AC de 0.82): con cinco ítems, es el autoconcepto general.

- Autonomía (AC de 0.88): con cinco ítems, son las oportunidades de disponer de su tiempo de ocio. 
- Relación con los padres y vida familiar (AC de 0.68): con seis ítems, es la atmósfera familiar.

- Amigos y ayuda social (AC de 0.81): con seis ítems, son las relaciones del chico con sus pares.

- Entorno escolar (AC de 0.86): con seis ítems, es la percepción del chico sobre su aptitud para el aprendizaje, la concentración y las sensaciones sobre la escuela.

- Aceptación social (bullying) (AC de $0.65)$ : con tres reactivos, son los sentimientos de rechazo por parte de los compañeros.

- Recursos económicos (AC de 0.83): con tres ítems, es la percepción sobre la capacidad financiera familiar.

Dando un total de 52 reactivos, se responde en una escala tipo Likert con cinco categorías de respuestas, cada una con un puntaje asignado, permitiendo obtener un puntaje que se traduce a una escala de 1 a 100 , en el que a mayor puntaje indica una mayor CVRS; de la misma manera se puede calificar cada dimensión por separado ${ }^{20-22}$.

\section{PROCEDIMIENTO}

Primero se acordó el plan de trabajo con los directivos escolares. Posteriormente se invitó a todos los adolescentes y a sus padres a participar en el proyecto y se les solicitó el consentimiento informado por escrito y el cuestionario de características sociodemográficas inicial con ayuda de los padres.

Luego, a los adolescentes que otorgaron el asentimiento verbal se les aplicaron entrevistas y cuestionarios autoaplicables; además, cada adolescente fue medido y pesado por personal previamente capacitado (dos médicos y una enfermera), de acuerdo a las recomendaciones del manual de antropometría del Instituto Nacional de Ciencias Médicas y Nutrición Salvador Zubirán, en dos básculas calibradas que tenían estadímetros.

\section{ANÁLISIS ESTADIÍSTICO}

Los datos recabados se consignaron en una base de datos Excel y se realizó un análisis estadístico en Minitab 4, que consistió en un análisis exploratorio a través de frecuencias, porcentajes y medidas de tendencia central, que permitió comparar al grupo de satisfechos con el de insatisfechos y así identificar las diferencias entre ambos grupos. Se construyó la razón de insatisfacción para el IMC dividiendo los insatisfechos entre el total de adolescentes. Para determinar si las diferencias eran significativas, se realizó un análisis a través de pruebas de chi cuadrada y $U$ de Mann Whitney; en las variables en las que se identificó significancia estadística con insatisfacción de la imagen corporal, se aplicó el coeficiente de correlación de Spearman.

\section{CONSIDERACIONES ÉTICAS}

Para la realización de este trabajo se obtuvo la aprobación de un comité local de investigación, otorgándosele el número 2015-3004-13 en el Sistema de Registro Electrónico de la Coordinación de Investigación en Salud del Instituto Mexicano del Seguro Social. Fue hecha con base en el artículo 17, fracción II, del Reglamento de la Ley General de Salud.

\section{RESULTADOS}

En el presente estudio se incluyeron un total de 437 adolescentes, obteniendo que el 69\% (303) de los adolescentes tiene insatisfacción de su imagen corporal y el 31\% (104) está satisfecho con su imagen.

Respecto al sexo, en ambos grupos predominaron las mujeres, con un 57\% para los adolescentes insatisfechos y un 58\% para los satisfechos. También predominó el grupo etario de 13-14 años, con un 63\% para los satisfechos y para los insatisfechos, sin presentar diferencias estadísticamente significativas; sin embargo, en cuanto al IMC, predominó el peso normal, con un $43 \%$ para los insatisfechos y un $75 \%$ para los satisfechos, cuya relación presentó valores de $\mathrm{p}<0.05$ (Tabla 1).

La distribución predominante para ambos grupos fue similar respecto a la mayoría de los factores estudiados, a excepción del IMC, en el que se determinó a través del índice de insatisfacción, que existe mayor insatisfacción en los adolescentes obesos, seguidos de 
Tabla 1. Relación entre IMC e insatisfacción de la imagen corporal en adolescentes

\begin{tabular}{|c|c|c|c|c|c|c|c|}
\hline \multirow{2}{*}{$\begin{array}{l}\text { Clasificación } \\
\text { por IMC }\end{array}$} & \multicolumn{2}{|c|}{ Global } & \multicolumn{2}{|c|}{ Insatisfechos } & \multicolumn{2}{|c|}{ Satisfechos } & \multirow{2}{*}{$\begin{array}{c}\text { Índice de } \\
\text { insatisfacción }\end{array}$} \\
\hline & $f x$ & $\%$ & $f x$ & $\%$ & $f x$ & $\%$ & \\
\hline Bajo peso & 5 & $1.1 \%$ & 4 & $1.3 \%$ & 1 & $0.8 \%$ & 0.80 \\
\hline Peso normal & 232 & $53.1 \%$ & 131 & $43.2 \%$ & 101 & $75.4 \%$ & 0.56 \\
\hline Sobrepeso & 120 & $27.5 \%$ & 91 & $30.0 \%$ & 29 & $21.6 \%$ & 0.76 \\
\hline Obesidad & 80 & $18.3 \%$ & 77 & $25.5 \%$ & 3 & $2.2 \%$ & 0.96 \\
\hline Total & 437 & $100 \%$ & 303 & $100 \%$ & 134 & $100 \%$ & 0.69 \\
\hline
\end{tabular}

$n=437$. chi cuadrada $=47.98 \cdot p=0.0000$.

Tabla 2. Factores psicosociales asociados con la insatisfacción de la imagen corporal en adolescentes

\begin{tabular}{|c|c|c|c|}
\hline $\begin{array}{l}\text { Dimensiones de la escala } \\
\text { de calidad de vida general (global) }\end{array}$ & $\begin{array}{c}\text { Insatisfechos } \\
(n=303) \\
\text { Puntaje }\end{array}$ & $\begin{array}{c}\text { Satisfechos } \\
\text { (n=134) } \\
\text { Puntaje }\end{array}$ & $\begin{array}{c}\text { Valores de p } \\
\text { (U-Mann Whitney) }\end{array}$ \\
\hline Bienestar físico & 64 & 72.0 & $0.0006^{*}$ \\
\hline Bienestar psicológico & 80 & 83.0 & 0.1551 \\
\hline Estado de ánimo & 80 & 81.4 & $0.0249^{\dagger}$ \\
\hline Autopercepción & 76 & 88.0 & $0.00001^{*}$ \\
\hline Autonomía & 64 & 72.0 & 0.1389 \\
\hline Relación con los padres/familia & 83.33 & 86.6 & 0.1278 \\
\hline Recursos económicos & 60 & 66.6 & 0.2380 \\
\hline Relación con los amigos/pares & 83.33 & 86.6 & $0.0198^{\dagger}$ \\
\hline Entorno escolar & 70 & 73.0 & 0.2388 \\
\hline Aceptación social/bullying & 93.33 & 93.3 & 0.1105 \\
\hline Puntaje total & 74 & 78.0 & $0.0010^{*}$ \\
\hline
\end{tabular}

$\mathrm{n}=437 .{ }^{*} \mathrm{p}<0.001 .{ }^{\mathrm{t}} \mathrm{p}<0.05$.

Nota: A mayor puntuación, mejor calidad de vida.

aquéllos con bajo peso y posteriormente con sobrepeso, siendo el índice de insatisfacción más bajo en los adolescentes con peso normal. Además, hubo relación estadísticamente significativa entre IMC e insatisfacción de la imagen corporal, con valores de $\mathrm{p}<0.01$.

De la misma manera para el caso de los factores biopsicosociales, en los que el bienestar físico, el estado de ánimo, la autopercepción y la relación con los pares/amigos presentaron una $\mathrm{p}$ significativa con valores $<0.05$ (Tabla 2).

Posteriormente, con los factores identificados como relacionados, se determinó la correlación con la insatisfacción de la imagen corporal por medio del coeficiente de correlación de Spearman como se indica en la tabla 3.

\section{DISCUSIÓN}

Los factores que se encontraron relacionados con la insatisfacción de la imagen corporal en adolescentes fueron, de los biológicos, el estado nutricional (IMC), y de los psicosociales, el bienestar físico, el estado de ánimo, la autopercepción y la relación con los pares/amigos. Esto confirma que el constructo de imagen corporal está influenciado por aspectos tanto inherentes al individuo como a su entorno, lo cual coincide con lo descrito previamente por diversos autores $5,72,23$.

Se determinó una elevada prevalencia de insatisfacción en adolescentes, a pesar de que la mayoría tiene peso normal. La prevalencia en los mismos grupos de edad es similar en poblaciones españolas y mexicanas; sin 
Tabla 3. Correlación de factores biopsicosociales con la insatisfacción de la imagen corporal en adolescentes

\begin{tabular}{|l|c|}
\hline $\begin{array}{l}\text { Factores relacionados } \\
\text { con la insatisfacción } \\
\text { de la imagen corporal }\end{array}$ & $\begin{array}{c}\text { Correlación } \\
\text { de Spearman (rho) }\end{array}$ \\
\hline IMC & 0.404 \\
\hline Bienestar físico & -0.211 \\
\hline Estado de ánimo & -0.149 \\
\hline Autopercepción & -0.400 \\
\hline Relación con los pares/amigos & -0.97 \\
\hline
\end{tabular}

n=437. IMC: índice de masa corporal.

embargo, en Brasil alcanzan a reportar alrededor del 20\%. La diferencia en estos datos pudiera ser por el tipo de instrumento para identificar la insatisfacción de la imagen con el Body Shape Questionary en Brasil y la escala de las siluetas de Stunkard en este trabajo, así como también por las características de la población -en el caso de los adolescentes mexicanos, con más sobrepeso y obesidad- $-^{7,8,15}$.

En lo referente a la relación de la imagen corporal con los factores biológicos, en este estudio no hubo relación entre la insatisfacción de la imagen corporal con el sexo, similar a lo reportado en Brasil y España ${ }^{3,12}$; lo mismo sucedió con la edad. Este hallazgo contrasta con otras investigaciones que indican que existen diferencias significativas entre hombres y mujeres, y que, conforme aumenta la edad, disminuye la insatisfacción con la imagen del cuerpo ${ }^{8,10-12,24}$.

Estas generaciones de adolescentes parecen estar en mayor riesgo de desarrollar actitudes poco saludables hacia sus cuerpos al basar su percepción en torno a la influencia de la publicidad, presión por mensajes verbales y modelos sociales ${ }^{12}$; incluso tienen acceso, cada vez a menor edad, a los nuevos medios de comunicación y redes sociales, en los que se impone un modelo de imagen preestablecido $^{5}$. La imposición a la mujer de la figura delgada como sinónimo de belleza llega a ser perversa, y algo semejante sucede con el hombre, a quien se le promueve una figura más atlética. De hecho, estudios en poblaciones de jóvenes preuniversitarios mexicanos llegan a reportar entre un 70 y $81 \%$ de insatisfacción, es decir, persiste la elevada incidencia en grupos de mayor edad $d^{5,12,25,26}$.

Indudablemente, existe relación entre el IMC y la percepción de la imagen, siendo esta correlación positiva y coincidiendo con lo ya reportado en la literatura médica ${ }^{5,7,8,11,25}$. Sin embargo, como muestra el índice de insatisfacción respecto al IMC en la tabla 1, la correlación no obedece a un gradiente lineal, es decir, que no sólo con el incremento de peso irá aumentando la insatisfacción de la imagen, sino que cualquier diferencia respecto al peso normal ocasionará insatisfacción de la imagen corporal, y cuanto más se aparte del intervalo percentilar considerado como normal, mayor será la insatisfacción de la imagen. Esto también hace disentir de la propuesta basada en que el bajo peso es un factor protector (sobre todo en mujeres) para que haya satisfacción de imagen corporal ${ }^{7,8}$.

La importancia de la distribución del IMC y la forma de correlacionarse con la insatisfacción de en esta investigación radica en que da la pauta para el diseño de posteriores estudios de intervención, ya que, en el caso de los adolescentes con bajo peso, sobrepeso y obesidad, podemos utilizar la inconformidad en la percepción de la imagen como motivador para modificar el estado nutricional y tratar de llevarlos a su peso normal a través de actividad física y abordaje nutricional, pero en aquéllos con insatisfacción y peso normal, será necesario utilizar estrategias ubicadas en el eje psicológico y/o social27.

Se hace especial énfasis en abordar el estado nutricional de la población adolescente porque refleja fielmente la situación actual en México, país que figura como primer lugar en obesidad infantil y que prevalece en la adolescencia, según datos de la Encuesta Nacional de Salud y Nutrición, los cuales indican que 1 de cada 3 adolescentes de entre 12 y 19 años presenta sobrepeso u obesidad $\left(\right.$ ENSANUT 2012) ${ }^{28}$. Es menester mencionar que en la presente investigación el $45 \%$ de los adolescentes está por encima del peso normal, y en lo referente a los adolescentes 
insatisfechos con su imagen, aproximadamente 6 de cada 10 se encuentran en el rango de sobrepeso u obesidad.

Es incuestionable la importancia de este hallazgo, puesto que el sobrepeso y la obesidad son precedentes de enfermedades cardiovasculares, diabetes, trastornos osteomusculares y algunos tipos de cáncer en adolescentes, según la Organización mundial de la Salud (OMS 2016) ${ }^{27}$, razones por las que se reportó oportunamente a los directivos escolares con sugerencias específicas en medidas de atención, propuestas de seguimiento e intervención. Además, aunque no fue objetivo de este estudio, por responsabilidad ética ante las características de la población, se reportó que en los adolescentes con sobrepeso y obesidad disminuye la calidad de vida relacionada con la salud (CVRS), relación identificada a través de un análisis con la prueba de Krusskal Wallis, determinando que existe una relación significativa entre el IMC y la CVRS, específicamente en las dimensiones de bienestar físico y autopercepción.

El diseño de las intervenciones integrales propuestas para la disminución de peso y la mejora de la satisfacción corporal deben estar basadas en actividad física, nutriológica y psicoconductual, de acuerdo con la Norma Oficial Mexicana para el tratamiento de la Obesidad y Sobrepeso. Se sugiere que el abordaje involucre a la familia, pues como se describe en el Estudio Longitudinal Nacional de la Salud del Adolescente, de la Universidad de Arizona: «El comportamiento alimentarioy de convivencia se aprende más en casa...», recomendando promover el enfoque sistémico-familiar para afrontar los problemas sanitarios actuales referentes a la adolescencia ${ }^{29,30}$.

Respecto a la insatisfacción de la imagen corporal y los factores psicosociales, se determinó que las dimensiones bienestar físico, estado de ánimo, autopercepción y relación con los pares o amigos están relacionadas con la insatisfacción de la imagen corporal, situación que repercute de manera directa en la calidad de vida de los adolescentes.

En cuanto a la relación con el bienestar físico, en coincidencia con Meza Peña, et al.
$(2013)^{10}$, quienes propusieron investigar la relación de la imagen corporal con la alteraciones afectivas, en el presente estudio se encontró asociación significativa entre la insatisfacción y el estado de ánimo (Tabla 3).

La insatisfacción está asociada con la autopercepción y con la relación entre pares o amigos, como menciona Mariscal Rodríguez $z^{5}$ quien además enfatiza sobre las burlas o acoso escolar. No obstante, en este estudio no hubo relación con acoso escolar, quizás porque en el plantel donde se realizó esta investigación los directivos habían establecido previamente medidas educativas para prevenir el bulliyng en los adolescentes.

La relevancia en lo referente a los factores psicosociales es porque en este trabajo se demostró una correlación negativa, es decir, a menor bienestar físico, estado de ánimo, autopercepción y relación con los pares/amigos, mayor fue la insatisfacción de la imagen corporal, la cual está íntimamente relacionada con actitudes alimentarias de riesgo; algunos autores hasta la consideran como precursora de los trastornos de la conducta alimentaria ${ }^{7,25}$, mientras que otros la asocian con una menor calidad de vida de los preadolescentes, en particular en las dimensiones de bienestar físico, bullying, entorno escolar, estado emocional y ánimo, autopercepción, autonomía y recursos económicos ${ }^{17}$.

Este estudio marca la pauta sobre la necesidad de mayores investigaciones referentes a la insatisfacción de la imagen corporal a través de los factores psicosociales que la enmarcan, plantear la utilización más amplia de la escala de las siluetas de Stunkard (aplicación que dura $1 \mathrm{~min}$ ) y privilegiar estudios de intervención integrales para adolescentes que incluyan: actividad física, estrategias psicoeducativas enfocadas a reestablecer el buen estado de ánimo y renovar la autopercepción de los adolescentes, medidas educativas para mejorar las relaciones entre pares, y también con los padres (a través del fortalecimiento de la comunicación efectiva), así como la redirección de estas personas en búsqueda de identidad hacia el autocuidado y promoción de valores entre los adolescentes. 
Se requiere personal capacitado en el abordaje integral a la salud y un manejo multidisciplinario, para lo cual el médico familiar, por su perfil profesional, bien podría ser la respuesta que, sin duda, brindaría un gran impacto biopsicosocial a la salud de la población.

\section{CONCLUSIONES}

Los factores correlacionados con la insatisfacción de la imagen corporal en adolescentes de una población mexicana son: el IMC (directamente) y el bienestar físico, estado de ánimo, autopercepción y relación con los pares/amigos (inversamente).

\section{FINANCIAMIENTO}

\section{Ninguno.}

\section{CONFLICTO DE INTERESES}

Los autores declaran no tener ningún conflicto de interés.

\section{AGRADECIMIENTOS}

A Issa Gil Alfaro, Hilda Mendoza Sánchez, Balkis Cruz Mendoza y Tania López Chima. Y al Consejo Nacional de Ciencia y tecnología y Programa Nacional de Posgrados de Calidad (CONACYT) por formarme en un Programa Nacional de Posgrados de Calidad (PNPC).

\section{BIBLIOGRAFÍA}

1. Vaquero Cristóbal R, Alacid F, Muyor JM, López Miñarro PA. Imagen corporal: revisión bibliográfica. Nutr Hosp. 2013;28(1):27-35.

2. Salaberria K, Rodríguez S, Cruz S. Percepción de la imagen corporal. Osasunaz. 2007;8:171-83.

3. Gómez Mármol A, Sánchez Alcaraz BJ, Mahedero Navarrete MP. Insatisfacción y distorsión de la imagen corporal en adoles centes de doce a diecisiete años de edad. Ágora para la EF y el Deport. 2013;15(1):54-63.

4. Sánchez Vázquez I, Rossano Bernal B. Insatisfacción corporal en hombres y mujeres de población rural. (Tesis doctoral) Estado de México: Universidad Autónoma del Estado de México, Facultad de Ciencias de la Conducta; 2012.

5. Mariscal Rodríguez GL. Influencias socioculturales asociadas a la percepción corporal en niño(a)s: una revisión y análisis de la literatura. Rev Mex Trastor Aliment. 2013;4:24-67.

6. López Pérez AM, Solé Burgós A, Cortés Moscowich I. Percepción de satisfacción-insatisfacción de la imagen corporal en una muestra de adolescentes de Reus (Tarragona). Zainak. 2008;30:125-46.

7. Santana MLP, Silva RCR, Assis AMO, Raich RM, Machado MEPC, Pinto EJ, et al. Factors associated with body image dissatisfaction among adolescents in public schools students in Salvador Brazil. Nutr Hosp. 2013;28(3):747-55.

8. Cordeiro Beling MT, Ribeiro Ferreira MF, Silveira Barros AF, Beling F, Silveira Barros AF, Alves Lamounier J. Alterações na imagem corporal entre adolescentes do sexo feminino e fatores asociados. Adolesc Saude. 2012;9(4):11-8.

9. Silva-Filho L, Rabelo-Leitão AC, Menezes Cabral RL, Knackfuss MI. Imagen Corporal, Actividad Física y Factores de Riesgo. Rev Salud Publica. 2008;10(4):550-60.
10. Meza Peña C, Pompa Guajardo E. Insatisfacción con la Imagen Corporal en Adolescentes de Monterrey. Daena International Journal of Good Conscience. 2013;8(1):32-43.

11. Trejo Ortiz PM, Castro Veloz D, Facio Solís A, Mollinedo Montano FE, Valdez Esparza G. Insatisfacción con la imagen corporal asociada al Índice de Masa Corporal en adolescentes. Revista Cubana de Enfermería. 2010;26(3):144-54.

12. Zaragoza Cortes J, Saucedo-Molina TJ, Fernández Cortés TL. Asociación de impacto entre factores socioculturales, insatisfacción corporal e índice de masa corporal en estudiantes universitarios de Hidalgo, México. Archivos Latinoamericanos de Nutrición. 2011;61(1):20-6.

13. Trujano P, Nava C, Gracia M, Limón G, Alatriste AL, Merino MT, et al. Trastorno de imagen: un estudio con preadolescentes y reflexiones desde la perspectiva de género. An Psicol. 2010;26(2):279-87.

14. Consejo Nacional de Población. Proyecciones de la población 2010-2050. México: Secretaría de Gobernación; 2014. Última modificación: viernes 25 de abril de 2014. Disponible en: http:// www.conapo.gob.mx/es/CONAPO/Proyecciones

15. López Chima TMC. Conductas alimentarias de riesgo y la insatisfacción de la imagen corporal en adolescentes de la zona de influencia de la UMF 66. (Tesis de especialidad). Xalapa, Veracruz: Universidad Veracruzana; 2013.

16. Stunkard A, Sorensen T, Schulsinger F. Use of the Danish Adoption resister for the study of obesity and thinness. Res Publ Assoc Res Nerv Ment Dis. 1983;60:115-20.

17. Urzúa MA, Avendaño HF, Díaz CS, Checura D. Calidad de vida y conductas alimentarias de riesgo en la preadolescencia. Rev Chil Nutr. 2010;37(3):282-92.

18. Urzúa MA, Cortés RE, Prieto C, Vega BS, Tapia NK. Autoreporte de la Calidad de Vida en Niños y Adolescentes Escolarizados. Rev Chil Pediatr. 2009;80(3):238-44.

19. Marcela Vélez C, García García HI. Medición de la calidad de vida en niños. latreia. 2012;25(3):240-9.

20. Berra Sivina, Tebé C, Eusandi ME, Carignano C. Fiabilidad y validez del cuestionario KIDSCREEN-52 para medir calidad de vida relacionada con la salud de la población argentina de 8-18 años. Arch Argent Pediatr. 2013;111(1):29-36.

21. Hidalgo Rasmussen CA, Rajmil L, Montaño Espinoza R. Adaptación transcultural del cuestionario KIDSCREEN para medir la calidad de vida relacionada con la salud en población mexicana de 8 a 18 años. Ciencia y Saúde Coletiva. 2014;19(7):2215-24.

22. Ravens-Sieberer U, Gosch A, Rajmil L, Erhart M, Bruil J, Dür W, et al. KIDSCREEN-52 medida de la calidad de vida de los niños y adolescentes. Revisión de Expertos de Farmacoeconomía y Resultados de Investigación. 2005;5(3):353-64. Disponible en: http://www.kidscreen.org/espa\%C3\%B1ol/

23. Guadarrama Guadarrama R, Carrillo Arellano SS, Márquez Mendoza O, Hernández Navor JC, Veytia López M. Insatisfacción corporal e ideación suicida en adolescentes estudiantes del Estado de México. Rev Mex Trastor Aliment/Mexican Journal of Eating Disorders. 2014;(5):98-106.

24. Fernández-Bustos JG, González-Martí I, Contreras O, Cuevas R. Relación entre imagen corporal y autoconcepto físico en mujeres adolescentes. Rev Latinoam Psicol. 2015;47(1):25-33.

25. Saucedo Molina TJ, Zaragoza Cortés J, Villalón L, Peña Irecta A León Hernández R. Prevalencia de factores de riesgo asociado a trastornos alimentarios en estudiantes universitarios hidalguenses. Psicología y Salud. 2015;25(2):243-51.

26. Soto Ruíz, Marín Fernández B, Aguinaga Ontoso I, Guillén-Grima F, Serrano Monzó I, Canga Armayor N, et al. Análisis de la percepción de la imagen corporal que tienen los estudiantes universitarios de Navarra. Nutrición Hospitalaria. 2015;31(5):2269-75.

27. Datos y cifras sobre obesidad infantil. Organización mundial de la Salud 2016. Consultado en enero de 2016. Disponible en: http://www.who.int/end-childhood-obesity/facts/es/

28. Gutiérrez JP, Rivera-Dommarco J, Shamah-Levy T, VillalpandoHernández S, Franco A, Cuevas-Nasu L, et al. Encuesta Nacional de Salud y Nutrición 2012. Resultados Nacionales. Cuernavaca, México: Instituto Nacional de Salud Pública, 2012.

29. Norma Oficial Mexicana NOM-008-SSA3-2010, Para el tratamiento integral del sobrepeso y la obesidad.

30. The National Longitudinal Study of adolescents to adult Health; Add Health. Consultado en enero de 2016. Disponible en: http:// www.cpc.unc.edu/projects/addhealth 